\title{
A HOMOGENEOUS, GLOBALLY SOLVABLE DIFFERENTIAL OPERATOR ON A NILPOTENT LIE GROUP WHICH HAS NO TEMPERED FUNDAMENTAL SOLUTION
}

\author{
DETLEF MÜLLER
}

(Communicated by Jonathan M. Rosenberg)

Dedicated to the memory of Lawrence Corwin

\begin{abstract}
We present an example of a homogeneous, left-invariant differential operator on the Heisenberg group $\mathrm{H}_{3}$ which admits fundamental solutions but no tempered ones. This answers a question raised by Corwin in the negative.
\end{abstract}

Assume that $N$ is a connected, simply connected nilpotent Lie group with Lie algebra $\mathfrak{n}$, and let $L \in \mathfrak{U}(\mathfrak{n})$ be a left-invariant differential operator on $N$. If $N$ is abelian, any such $L$ can be considered as a constant coefficient differential operator on some Euclidean space $\mathbb{R}^{n}$ and, by the theorem of Malgrange and Ehrenpreis (see [H2]), has a fundamental solution $F \in \mathscr{D}^{\prime}\left(\mathbb{R}^{n}\right)$, i.e., $L F=\delta$, where $\delta$ denotes the point measure at the identity. In fact, it was proved later by Hörmander [H1] and Lojasiewicz [L] that one can even find a tempered fundamental solution $F \in \mathscr{S}^{\prime}\left(\mathbb{R}^{n}\right)$.

The situation becomes drastically different if $N$ is nonabelian, since then there exist many operators in $\mathfrak{U}(\mathfrak{n})$ which are not even locally solvable. Assume in the sequel that $N$ admits a one-parameter family $\left\{\delta_{r}\right\}_{r>0}$ of automorphic dilations (see [FS]) and that $L$ is homogeneous, i.e., $L\left(\varphi \circ \delta_{r}\right)=r^{m}(L \varphi) \circ \delta_{r}$ for some $m>0$ and every $\varphi \in \mathscr{D}(N), r>0$. Then it is at least true that various notions of solvability coincide for $L$. For instance, $L$ is locally solvable at some point of $N$ if and only if $L C^{\infty}(N)=C^{\infty}(N)$, if and only if $L$ has a fundamental solution $F \in \mathscr{D}^{\prime}(N)$ (see, e.g., [B, M1]). Moreover, if $L^{T}$, the transpose of $L$, is hypoelliptic, then the same is true of the operator $L L^{T}$, as can be seen by Helffer-Nourrigat's theorem [HN], and one can make use of the homogeneity of $L$ in order to prove that $L L^{T}$, hence also $L$, has even a tempered fundamental solution $[\mathrm{F}, \mathrm{G}]$.

So, a natural question, which seems to have been open hitherto, is whether any solvable, homogeneous, left-invariant differential operator or, more generally, every globally solvable left-invariant differential operator on a nilpotent Lie group has a tempered fundamental solution. In the latter form, this question was raised by Corwin in [C].

The purpose of this note is to present an example on the 7-dimensional

Received by the editors October 31, 1991 and, in revised form, August 26, 1992.

1991 Mathematics Subject Classification. Primary 22E30, 35A08. 
Heisenberg group $\mathrm{H}_{3}$ which answers this question in the negative, even for homogeneous operators.

Namely, if $X_{1}, X_{2}, X_{3}, Y_{1}, Y_{2}, Y_{3}, U$ denotes the standard basis of the Lie algebra $\mathfrak{h}_{3}$ of $H_{3}$ (with nontrivial brackets $\left[X_{j}, Y_{j}\right]=U, j=1,2,3$ ), we set

$$
L=\left(X_{1}^{2}+Y_{1}^{2}\right)-\lambda\left(X_{2}^{2}+Y_{2}^{2}\right)+Y_{3}^{2}, \quad \lambda \in \mathbb{R} \backslash\{0\} .
$$

Adopting the notation used throughout [MR1, MR2, M2], we have $L=\triangle_{S}$, where $S \in \mathfrak{s p}(3, \mathbb{R})$ is given by the matrix

$$
S=\left(\begin{array}{cc}
H & 0 \\
0 & N
\end{array}\right), \quad H=\left(\begin{array}{cccc}
0 & 0 & -1 & 0 \\
0 & 0 & 0 & \lambda \\
1 & 0 & 0 & 0 \\
0 & -\lambda & 0 & 0
\end{array}\right), \quad N=\left(\begin{array}{cc}
0 & 0 \\
1 & 0
\end{array}\right)
$$

with respect to the basis $X_{1}, X_{2}, Y_{1}, Y_{2}, X_{3}, Y_{3}$. Since $S$ is not semisimple, $L$ is locally solvable for every $\lambda \in \mathbb{R}$ by [MR2, Theorem (i.3)].

Now assume there is some $F \in \mathscr{S}^{\prime}\left(H_{3}\right)$ such that $L F=\delta$. If $(z, u)$, with $z \in \mathbb{R}^{6}, u \in \mathbb{R}$, denote the usual coordinates of $H_{3}$, we define as in [MR1]

$$
\mathscr{F}_{u}(z, \mu):=f^{\mu}(z):=\int_{\mathbb{R}} f(z, u) e^{-2 \pi i \mu u} d u, \quad \mu \in \mathbb{R},
$$

for $f \in \mathscr{S}\left(H_{3}\right)$. This partial Fourier transform turns $L$ into the "twisted" differential operator $\tilde{L}^{\mu}$ given by the formula

$$
(L f)^{\mu}=\tilde{L}^{\mu} f^{\mu},
$$

if $f \in \mathscr{S}\left(H_{3}\right)$. Let $\delta_{r}(z, u)=\left(r z, r^{2} u\right)$ denote the usual dilations on $H_{3}$, and fix a real function $\chi \in C_{0}^{\infty}\left(\mathbb{R}^{+}\right)$with support contained in the interval [1, 2] and $\int \chi(r) d r=1$. For $\varphi \in \mathscr{S}\left(\mathbb{R}^{6}\right)$ and $j=0,1$, we set

$$
A_{j} \varphi(z, u):=\int_{0}^{\infty} \varphi\left(r^{1 / 2} z\right) e^{-2 \pi i r u} \chi(r) r^{j} d r .
$$

Then $A_{j}$ defines a continuous linear operator from $\mathscr{S}\left(\mathbb{R}^{6}\right)$ into $\mathscr{S}\left(H_{3}\right)$, a fact which follows easily from the formula

$$
A_{j} \varphi=\mathscr{F}_{u}\left(E_{j} \varphi\right),
$$

where $E_{j} \varphi$ is defined by

$$
E_{j} \varphi(z, \mu):=\varphi\left(\mu^{1 / 2} z\right) \chi(\mu) \mu^{j} .
$$

Moreover, from (3) one easily sees that

$$
L \mathscr{F}_{u}\left(E_{0} \varphi\right)=\mathscr{F}_{u} E_{1}\left(\tilde{L}^{T} \varphi\right) ;
$$

hence

$$
L A_{0} \varphi=A_{1} \tilde{L}^{T} \varphi
$$

where we have set $\tilde{L}:=\tilde{L}^{1}$. 
Let $A_{1}^{T}: \mathscr{S}^{\prime}\left(H_{3}\right) \rightarrow \mathscr{S}^{\prime}\left(\mathbb{R}^{6}\right)$ denote the adjoint operator to $A_{1}$, and let $K \in \mathscr{S}^{\prime}\left(\mathbb{R}^{6}\right)$ be given by $K=A_{1}^{T}(F)$. Then, by (4),

$$
\begin{aligned}
\langle\tilde{L} K, \varphi\rangle & =\left\langle K, \tilde{L}^{T} \varphi\right\rangle=\left\langle F, A_{1} \tilde{L}^{T} \varphi\right\rangle=\left\langle F, L A_{0} \varphi\right\rangle \\
& =\left\langle L F, A_{0} \varphi\right\rangle=\left(A_{0} \varphi\right)(0),
\end{aligned}
$$

since $L=L^{T}$. Moreover, since

$$
\left(A_{0} \varphi\right)(0)=\varphi(0) \int_{0}^{\infty} \chi(r) d r=\varphi(0),
$$

we see that $\tilde{L} K=\delta$, i.e., we have proved the following

Lemma 1. Assume L (given by (1)) has a tempered fundamental solution. Then the same is true of $\tilde{L}$.

Finally, we can invoke [M2] in order to prove

Proposition 2. Let $L$ be given by (1). Then:

(i) $L$ has a fundamental solution $F \in \mathscr{D}^{\prime}\left(\mathrm{H}_{3}\right)$ for every $\lambda \in \mathbb{R}$.

(ii) If $L$ has a tempered fundamental solution $F \in \mathscr{S}^{\prime}\left(H_{3}\right)$, then there are constants $C>0, r \in \mathbb{N}$ such that

$$
|\lambda-p / q|>C q^{-r}
$$

whenever $p$ and $q$ are odd positive integers such that $\lambda-p / q>0$. In particular, $L$ has no tempered fundamental solution, if $\lambda=\lambda_{0}$, where $\lambda_{0}:=\sum_{k=0}^{\infty} 3^{-k !}$. Proof. It has been shown in [MR2, Proposition 3.9] that the Liouville number $\lambda_{0}$ violates condition (5), so there remains only to prove (ii).

But, in the notation of [M2], the matrix $S$ associated to $L$ is of type $(E 1)$, and condition (5) is equivalent to [M2, Theorem 1.1, condition (1.8)]. Therefore, by [M2, Corollary 3.2 and Theorem 1.1], $\tilde{L}$ can have a tempered fundamental solution only if (5) holds; hence (ii) follows from Lemma 1. Q.E.D.

Remark 3. In [M1] we showed that a homogeneous operator $L \in \mathfrak{U}(\mathfrak{n})$ is not locally solvable if there is a sequence $\left\{\psi_{j}\right\}_{j} \subset \mathscr{S}(N)$ such that $\psi_{j}(0)=1$ for every $j$ and

$$
\lim _{j \rightarrow \infty}\left\|\psi_{j}\right\|_{(N)}\left\|L^{T} \psi_{j}\right\|_{(N)}=0
$$

for every Schwartz-norm $\|\cdot\|_{(N)}$. This condition relaxes the necessary condition for local solvability in [CR] and was crucial in [MR2] but may look somewhat unnatural. One is tempted to ask if (6) could be replaced by

$$
\lim _{j \rightarrow \infty}\left\|L^{T} \psi_{j}\right\|_{(N)}=0 .
$$

However, Proposition 2 implies that this is not possible, for, if we could replace (6) by (7), then local solvability of $L$ would imply an estimate of the form

$$
|\psi(0)| \leq\left\|L^{T} \psi\right\|_{(N)}, \quad \psi \in \mathscr{S}(N),
$$

for some Schwartz-norm $\|\cdot\|_{(N)}$. And, by the Hahn-Banach theorem, this would mean that $L$ had a tempered fundamental solution.

\section{ACKNOWLEDGMENT}

I wish to thank the referee for several useful comments on the paper. 


\section{REFERENCES}

[B] F. Battesti, Résolubilité globale d'opérateurs différentiels invariants sur certains groupes de Lie, J. Funct. Anal. 77 (1988), 261-308.

[C] L. Corwin, Criteria for solvability of left-invariant operators on nilpotent Lie groups, Trans. Amer. Math. Soc. 280 (1983), 53-72.

[CR] L. Corwin and L. P. Rothschild, Necessary conditions for local solvability of homogeneous left invariant differential operators on nilpotent Lie groups, Acta Math. 147 (1981), 265-288.

[F] G. B. Folland, Subelliptic estimates and function spaces on nilpotent groups, Ark. Mat. 13 (1975), 161-207.

[FS] G. B. Folland and E. M. Stein, Hardy spaces on homogeneous groups, Princeton Univ. Press, Princeton, NJ, 1982.

[G] D. Geller, Liouville's theorem for homogeneous groups, Comm. Partial Differential Equations 8 (1983), 1655-1677.

[H1] L. Hörmander, On the division of distributions by polynomials, Ark. Mat. 3 (1958), 555-568.

[H2] Linear partial differential operators, Springer, New York, 1964.

[HN] B. Helffer and J. Nourrigat, Caractérisation des opérateurs hypoelliptiques homogènes invariants à gauche sur un groupe de Lie nilpotent gradué, Comm. Partial Differential Equations 4 (1979), 899-958.

[L] S. Lojasiewicz, Sur le problème de division, Studia Math. 18 (1959), 87-136.

[M1] D. Müller, A new criterion for local non-solvability of homogeneous left-invariant differential operators on nilpotent Lie groups, J. Reine Angew. Math. 416 (1991), 207-219.

[M2] _ Local solvability of first order differential operators near a critical point, operators with quadratic symbols and the Heisenberg group, Comm. Partial Differential Equations 17 (1992), 305-337.

[MR1] D. Müller and F. Ricci, Analysis of second order differential operators on Heisenberg groups. I, Invent. Math. 101 (1990), 545-582.

[MR2] _ Analysis of second order differential operators on Heisenberg groups. II, J. Funct. Anal. 108 (1992), 296-346.

Fakultät für Mathematik, Universität Bielefeld, D-4800 Bielefeld 1, Germany Current address: Département de Mathématiques, Université Louis Pasteur, 7, rue René Descartes, F-67084 Strasbourg Cedex, France

E-mail address: dmuller@math.u-strasbg.fr 